\title{
DINI institutional repository certification and beyond
}

\author{
Susanne Dobratz \\ Humboldt-University, Berlin, Germany \\ Frank Scholze \\ Stuttgart University Library, Stuttgart, Germany
}

\begin{abstract}
Purpose: Overview on certification of institutional repositories as a means to support Open Access in Germany and description of the DINI Certificate 2006 developed by DINI, the German Initiative for Networked Information.

Design/methodology/approach: The DINI Certificate for Document and Publication Repositories shows potential users and authors of digital documents that a certain level of quality in operating the repository is guaranteed and that this distinguishes it from common institutional web servers. The Certificate can also be used as an instrument to support Open Access.

Findings: Repository certification will not be the main factor in achieving open access to academic information globally, but it can support the spread of institutional repositories and enhance visibility of the "Institutional Repository"-service.

Research limitations/implications: The DINI Certificate as a "soft" certificate aims towards interoperability of digital repositories, the coaching idea prevails. It does not provide an exhaustive auditing tool for trusted digital long term preservation archives.

Practical implications: The DINI Certificate for Document and Publication Repositories pushed the development of institutional repositories in Germany according to certain organisational and technical standards and contributes to the interoperability amongst digital repositories worldwide.
\end{abstract}

Originality/value: This paper describes a unique approach that has been implemented in Germany and could be transferred to other countries and communities.

Keywords: Digital storage, Archives management, Germany

Paper type: Conceptual paper

\section{Introduction: about DINI}

DINI [1], the German Initiative for Networked Information (Deutsche Initiative für Netzwerkinformation) is a coalition formed by German Higher Education infrastructure and service institutions, such as libraries, computing centres and media centres, as well as by scientific learned societies. DINI itself is not a funding body, but it co-operates with German funding agencies like the German Research Foundation (DFG) or the German Ministry for Education and Research (BMBF).

DINI's primary objective is to create recommendations for standardized and interoperable information services and communication networks in and between universities. Therefore, the structuring and construction of networked digital publication services is one major task. DINI does this by evaluating and disseminating examples of good or best practise and by initiating and intensifying regional, national and international collaboration. International ideas, developments, and technologies, such as the Open Archives Initiative Protocol for Metadata Harvesting (OAI-PMH), are observed and adapted for use in Germany. In this case the DINI Electronic Publishing Working Group formulated usage 
guidelines for institutional repositories in Germany (DINI Electronic Publishing Working Group, 2005).

DINI's focus is therefore the development and support of an information infrastructure. DINI is accomplishing this through operating a number of working groups [2].

The DINI Electronic Publishing Working Group is the most active group within DINI, having started its activities immediately after DINI was established in 2000. The members come from German universities and Higher Education institutions. Notable activities of the group were the organisation of workshops, such as the OAl Tutorials in Germany in 2003, the Workshop on the Implementation of Digital Repositories in Frankfurt in 2004 or the Open Access Symposium [3] in Göttingen in 2005. This event marked the beginning of Open Access activities at German universities. It was followed by the International Workshop on Institutional Repositories and Enhanced and Alternative Metrics of Publication Impact in Berlin, 2006 [4], which focused on issues of visibility and the impact of scientific publications.

\section{Certification in the DINI context}

The DINI Certificate for Document and Publication Repositories (DINI Electronic Publishing Working Group, 2003) distinguishes the repository from common institutional web servers and assures potential users and authors of digital documents that a certain level of quality in repository operation and services is guaranteed.

The primary objective of the guidelines and criteria is:

- to improve interoperability and co-operation between German Higher Education institutions that run digital repositories; and

- $\quad$ to provide a tool for repository operators that could be used to raise the visibility, recognition, and importance of the digital repository within the university.

In addition, DINI sees its certificate as a tool for supporting the Open Access concept. It is regarded as a "soft certificate" (Dobratz and Schoger, 2005), focusing on the concept of coaching. Thus DINI defines certification slightly differently from others, who focus more on long-term preservation aspects (Dale, 2005; Ross and McHugh, 2005). One of the initiatives working on an auditing tool for certifying trusted digital repositories in the context of long term preservation is the RLG/NARA (Research Libraries Group / National Archives and Records Administration) Task Force on Digital Repository Certification (see RLG/NARA Task Force on Digital Repository Certification, 2005; RLG Working Group on Digital Archive Attributes, 2002). Also nestor [5], the Network of Expertise in LongTerm Storage of Digital Resources for Germany (Dobratz et al., 2005), has established a working group on trusted repository certification, that is working on issuing a list of criteria for trusted digital long-term preservation repositories. The RLG/NARA audit checklist and the nestor certificate aim to document the trustworthiness of digital repositories.

Trustworthy digital repositories as defined by nestor can assure authors of all kinds of digital objects that their content is secured and preserved in a manner that ensures their authenticity and data integrity. It also provides a certain confidence for end users that the information will be accessible over time and that 
the objects received from the digital repository are trustworthy in terms of the authenticity of the objects, the author, and publication time and place. For the institution itself and its co-operating partners, the certificate guarantees the reliability of the digital archiving services, which is a prerequisite for its integration into the overall mission of the institution and for collaboration on a national or international level.

\section{DINI Certificate for Document and Publication Repositories}

The DINI Certificate aims at networking document and publication repositories by promoting the use of standards, interoperability and cooperation between German Higher Education institutions running digital repositories. By installing and running document and publication repositories, universities are able to offer and to archive scholarly publications that have been produced in-house, and make them available to a world-wide audience. This new service offered by the universities' information infrastructure units helps to disseminate the concept of electronic publishing as a new tool for academic work (Schirmbacher, 2005).

Since 1997, digital repositories have been developed in Germany with funding from the German Research Foundation (DFG) or the German Ministry of Education and Research (BMBF) (Scholze and Stephan, 2002). The reason for initiating a certificate for repositories was the result of a survey, which was conducted in 2003 by members of the DINI Electronic Publishing Group. 47 German universities answered questions regarding the technology and standards used for their document servers. Summarizing the results, a very disappointing picture was drawn concerning the use of standards for metadata, interfaces and cataloguing. In order to establish a German infrastructure for document repositories, DINI worked out criteria and guidelines; the DINI Certificate for Document and Publication Repositories. With this certificate DINI provides a tool for repository operators that can be used to raise the visibility, recognition and importance of the digital repository within the university.

The certificate shows potential users and authors of digital documents that a certain level of quality in operating the repository is guaranteed, and that this distinguishes it from common institutional web servers. The DINI criteria are split into two sections. The first section specifies minimum standards and requirements that must be met by the document and publication repositories or their operators in order for them to be awarded the certificate. The recommendations are, as far as we can judge today, likely to become future requirements for the certificate. The auditing process is based on self-disclosure by the repositories and is conducted by two domain experts who are announced by the DINI office for each individual certification process.

The requirements and recommendations cover the following topics:

- visibility and server policy,

- $\quad$ author support,

- legal issues,

- authenticity and integrity,

- $\quad$ indexing (subject indexing, metadata, interfaces),

- visibility / impact / access statistics,

- long-term availability. 
A working group within DINI audits the criteria for the DINI Certificate against international standards and developments and updates them accordingly. This continuous adaptation of the recommendations and requirements will ensure that the certificate retains its validity in an ever-changing environment.

For this reason the certificate is issued with a year-of-award stamp. The DINI office is responsible for awarding the DINI Certificate - the document that acknowledges that the certified repository meets the minimum standards of a DINI Certified Document and Publication Repository. A small fee (50 - 250 Euro) is charged for issuing the DINI Certificate.

\section{Practical experiences}

So far, 17 university repositories have been awarded the DINI Certificate [6], 2 repositories are currently being audited. It is often reported that the certification procedure has caused local authorities to reflect more deeply about the repository service itself and to start thinking about the repository's mission and philosophy. The certification recommendations and guidelines have proved to be a good way to bring the local repository up to a certain level of quality and to bring this to the attention of the institution's management.

Within the minimum requirements the following issues seem to be the most difficult, as has been reported by the operators of certified repositories:

- $\quad$ introduction of a server policy, because the institution's governing body has to be involved;

- $\quad$ provision of a visible service for authors;

- $\quad$ implementation of persistent identifiers, such as the URN:NBN schema [7] used in Germany and provided by Die Deutsche Bibliothek, the German national library (Schroeder, 2003).

\section{Supporting Open Access through the DINI Certificate 2006}

The second issue of the certificate, the DINI Certificate 2006 (DINI Electronic Publishing Working Group, 2006), stays abreast of changes and focuses on giving guidance for institutional repository operators to help them to support the Open Access concept and in particular to position their repository within the "green way" (Harnad, 2001), but it also gives recommendations for supporting the "golden way to Open Access" (Guédeon, 2004; Harnad, 2005).

In particular, it concentrates on the service an institutional repository, as an organisational and technological unit, can offer to support local academics in supplying pre- and post-prints of journal articles, that have already been published in scientific journals.

The following paragraphs describe the ideas for the new DINI Certificate 2006. They are still subject to change within the ongoing editing process:

\section{Visibility and server policy}

In order to enhance the visibility of a document and publication repository, the range of services offered must be accessible via a single WWW-based entry point and a reference from the institution's home page to the repository's main entry point must exist. It is also recommended that the repository should be registered 
with an aggregating service, such as the Institutional Archives Registry [8] or the Directory of Open Access Repositories - OpenDOAR [9].

There are also requirements on the server policy. It must state which standards are provided for publications in the repository with regard to content and functional and technical quality. An archiving guarantee for defined time spans, depending on the content and the functional as well as technical quality of the publications, has to be defined, as well as procedures for the operation of the repository. In particular, the services that the operator of the document repository offers to authors and editors must be published.

A DINI certified repository must define an Open Access policy containing a clear commitment to support the "green way" to Open Access.

\section{Author support}

It is vital that consultancy services and support for local authors (academics) should be offered via web pages, e-mail and telephone, as well as person-toperson support. Such support should be given for the entire publication process (including technical and legal areas).

In order to support the "green way" to Open Access the repository service must issue action guidelines for authors regarding secondary publications, provide self-upload for pre- and postprints and, as a minimum, guide the authors with a link to the SHERPA/ROMEO list on publisher's policies [10].

It is recommended that a curriculum of courses on electronic publishing should be offered at least once per semester, as well as specialized courses on "structured writing" for authors. The utilization of (electronic) help-desk systems and the provision of English language interfaces and descriptions may improve the quality and efficiency of author support.

The supply of style sheets or templates, of help texts that can be downloaded by authors (e.g. to produce PDF files), of references on how to use and cite documents as well as the provision of references to intellectual property rights and copyright is recommended.

\section{Legal issues}

The requirements for repositories are as follows. The operator of the repository must be permitted:

- to publish the uploaded document in the repository,

- to forward the document to an archiving institution,

- $\quad$ to alter the documents technically to secure long-term availability.

In addition, exemption from liability must be formulated in a disclaimer, and the operator must openly demonstrate, that the documents are protected by intellectual property rights, copyright or licenses.

Furthermore it is recommended that rights and privileges should be listed in the metadata, as has been done at the University of Tübingen [11], and that authors should be offered a licensing tool during the upload process, as is done with Creative Commons [12] and additional licenses at the University of Tübingen with the local institutional repository TOBIAS-lib. 


\section{Authenticity and data integrity}

In terms of ensuring a certain security, there are firstly requirements aiming to support the security of the repository. These include the documentation of the technical system (it is expected that the repository will be available 24 hours a day for seven days a week), the provision of a reliable back-up system to secure the repository, metadata and documents, and the existence of mechanisms that allow a technically controlled and verifiable acceptance of documents. In addition, the use of standard security mechanisms like SSL certification is recommended.

Secondly, the security of the documents has to meet a certain level. This means assigning persistent identifiers like Uniform Resource Names (URN) or Digital Object Identifiers (DOI) to the documents. It also means that a document with altered content must be treated as a new document, and therefore receive a new persistent identifier. Archiving the authors' uploaded files in their original format is also mandatory for a DINI certified repository. Recommendations for securing the documents themselves are the usage of an advanced digital signature technology, as provided by some vendors in Germany like Telesec [13], D-Trust [14] or Signtrust [15] ,according to the German Digital Signature Act (2005), or procedures to control the integrity of documents, such as hash algorithms.

Thirdly, in order to ensure the long-term availability of the documents, it is highly recommended that archiving file formats be rendered in order to export documents into long-term archiving facilities or institutions, such as kopal (Cooperative Development of a Long-Term Digital Information Archive) [16].

\section{Indexing (subject indexing, metadata, interfaces)}

Measures for enhancing the visibility of the documents and the servers include metadata, subject and formal indexing as well as interfaces for metadata exchange. As there are national classification schemas (e.g. the Regensburger Verbundklassifikation / RVK [17]) and normalized vocabulary for subject indexing terms (Schlagwortnormdatei / SWD [18]), DINI recommends, or even requires, the usage of these in order on the one hand to support a structured metadata exchange between the repositories, and, on the other hand, to enable libraries to treat their electronic publications like all other material held in their catalogues. By applying common library rules to the document and publication repositories, they are introduced into the usual library workflow and therefore receive more recognition by the library staff themselves. As digital publications are normally maintained within separate systems, using special repository software like EPrints.org [19], D-Space [20] or OPUS [21], which, by default, are not automatically integrated into Online Public Access Catalogues (OPAC) or library network catalogues, it has to be actively and deliberately decided by the repository operators to use the appropriate features. E.g. OPUS 3.0 uses an adapted OAIinterface to exchange enriched XMETADISS data with the union catalogue of the South-West German Library Network (SWB).

For subject indexing, DINI requires:

- the availability of a defined policy which should be made known to authors,

- the usage of verbal indexing with keywords or classificatory indexing, and

- the use of the Dewey Decimal Classification (DDC) according to usage in the German National Bibliography [22] as the general classificatory indexing 
system for documents (see also DINI Electronic Publishing Working Group, 2005).

Recommendations covering subject indexing are: the use of at least one additional standardized system of verbal or classificatory indexing (general or subject specific), e. g. Schlagwortnormdatei (SWD), Library of Congress Subject Headings, ACM Computing Classification System (CCS), Mathematical Subject Classification (MSC), or Physics and Astronomy Classification Scheme (PACS). In order to make German documents internationally accessible and searchable, the provision of keywords in English and abstracts in German and English is considered important.

The following issues regarding the use and availability of metadata are required: Firstly, the metadata must be available for free, and secondly as a minimum, the metadata should be encoded as Unqualified Dublin Core (ISO, 2003) according to OAI-PMH (DINI Electronic Publishing Working Group, 2005; Lagoze et al., 2002).

As a recommendation for metadata, DINI suggests the use of Qualified Dublin Core, ONIX (Online Information eXchange) [23], and the enhancement of metadata with technical and archival metadata, using standards like Metadata Encoding and Transmission Standard (METS), Long-term Preservation Metadata for Electronic Resources (LMER) or PREMIS (PREservation Metadata: Implementation Strategies) (see Die Deutsche Bibliothek, 2006; METS Editorial Board, 2005; PREMIS Working Group, 2005). Also recommended is the enhancement of metadata with special service metadata like print-on-demand data, e.g. PROPRINT (see Mittler and Schulz, 2004).

It is also highly recommended that metadata and documents should be able to be exported to long-term archiving institutions or repositories and that metadata should be able to be exported to bibliographical databases (e. g. bibliographical management system, library networks, OPACs).

In order to enhance the availability of the documents via services like Google or SCIRUS [24], or via scholarly search engines like BASE (Bielefeld Academic Search Engine) [25], DINI suggests the provision and promotion of special link lists for indexing by robots and commercial search engines.

Moreover, a DINI certified repository provides a WWW end-user interface and an OAI-PMH 2.0 interface in accordance with the DINI Recommendations on OAI-PMH Use in Germany (DINI Electronic Publishing Working Group, 2005).

It also uses extended metadata schemas with OAI 2.0 to allow for the exchange of complex metadata schemata and additional web service interfaces (e. g. Simple Object Access Protocol / SOAP) on a voluntary basis. The use of a Z39.50 interface is also recommended as it gives the chance of integrating the repository with library networks, which mainly operate on this particular protocol.

\section{Visibility - impact - access statistics}

As there are intensive discussions on introducing a way of measuring the impact and use of electronic documents in institutional repositories, DINI sees the necessity of helping repository operators to introduce such a technology, once it has been approved. Possible technologies and approaches are discussed, amongst others, by Bollen et al. (2005), Brody (2006), Hardy et al. (2005), Harnad et al. (2004), and Lawrence (2001). 
A prerequisite for providing reliable usage statistics are normalized webserver log files. A recommendation that is likely to become a standard in the next edition of the DINI Certificate is the use of the COUNTER (Counting Online Usage of Networked Electronic Resources) model for processing access logs from electronic journals or e-books. The COUNTER model cannot, at present, be assigned to all institutional repositories and the material available there in the same way, because issues like web crawler access have not yet been resolved. The DINI Electronic Publishing Working Group dedicates itself to supporting the development of such a model in co-operation with COUNTER and other interested parties like project IRS (Interoperable Repository Statistics) (see COUNTER, 2006; COUNTER, 2005). For the time being DINI recommends the use of existing lists to filter non-human access [26].

The requirements demanded by DINI are as follows: each individual repository must (within the limits of the law) log statistical data on access to both the repository and to individual publications. The webserver logs have to be anonymized before they are stored for long periods of time.

In order to make usage visible to users and authors, it is necessary to link individual publications with their access statistics as dynamic metadata.

It is essential that access statistics be accompanied by documentation explaining which criteria were used to collect the basic data and how it was processed. Furthermore, it has to be indicated on the web pages that access figures that are not collected and processed in a universal and standardized way cannot be used to compare different repositories. Access figures, as they are published by the repositories today, can only be used to compare the access figures for documents in the same repository.

\section{Long-term availability}

Long-term preservation has become an important issue within the electronic publishing process. However, it is neither necessary nor possible, from an economic point of view, to turn every institutional repository, or every document and publication repository, into a trusted digital long-term preservation archive. This highlights the need for global co-ordination of institutional repositories on the one hand, and trusted digital archives on the other hand. This co-ordination demands the preparation of digital documents and their metadata as Submission Information Packages according to the Open Archival Information System (OAIS) (CCSDS, 2002).

DINI supports this concept and requires the persistent linking of metadata and documents (e. g. via a persistent identifier, or the storage of the metadata and document in one single container), the inclusion in the repository's policy that the minimum availability of a document be no less than five years, and the provision of archive copies that are free of digital rights management (DRM) measures, which prevent the use of long-term preservation strategies (migration, emulation).

Recommendations for the support of the long-term availability of digital documents are: to take steps to secure long-term availability, where necessary through co-operation with an archiving institution, and to support and promote the use of open file formats for long-term preservation (e.g. PDF/a instead of PDF).

The production of technical metadata for long-term preservation (e.g. using tools like the JSTOR / Harvard Object Validation Environment / JHOVE) is 
regarded as important preparation for the later execution of preservation strategies. This should be connected with the unique identification of the used file format in the metadata, with reference to public file format registries.

\section{Conclusion}

Repository certification by itself can certainly not accomplish the Open Access idea within universities and research institutions. As long as scholars and scientists continue to fail to adopt the Open Access concept, universities will not succeed in implementing Open Access, even if they were to offer perfectly functioning institutional repositories from a technological point of view.

It is therefore essential to promote the scholarly relevance of the institutional repository in order to reach a critical mass of documents. The most convincing factor in encouraging authors to follow Open Access is acknowledgement by colleagues. This is achieved by counting and measuring usage and citation data of publications. It has already been shown that for most disciplines the impact factor is increased if articles are available through Open Access (Harnad et al., 2004; Lawrence, 2001). These findings have to be publicised among academics. Another potential approach to advance on the "green" as well as the "golden road" to Open Access is to establish impact factors that take into account Open Access publications and to give them the same position as existing metrics like the ISI Impact Factor (IF) [27].

There are interesting suggestions for alternative metrics of impact and usage as has been shown in the DFG/DINI workshop on impact measures [4] in February 2006 (see also Ball, 2006). DINI will discuss these suggestions taking into account the basic data that has to be collected as well as the methods and algorithms applied to that data. DINI will implement selected approaches in a German "testbed" consisting of DINI certified servers.

In summary, repository certification will not be the main factor in achieving open access to academic information globally, but it can facilitate the spread of institutional repositories and enhance visibility of the "Institutional Repository" service.

It is obvious from DINI's perspective, that universities and research institutions need guidance in installing institutional repositories. Institutions need to reach their academic staff, as they are the authors, and promote the quality and add-on functionalities that can be obtained by using an institutional repository instead of a personal home page.

DINI, in co-operation with other German players such as the German Rectors' Conference (HRK), the Standing Conference of the Ministers of Education and Cultural Affairs of the Länder in the Federal Republic of Germany (KMK), the German Research Foundation (DFG) and the German Ministry of Education and Research (BMBF), will engage in providing information space, support for post- and pre-print issues, and guidance on Open Access policies and copyright issues for German Higher Education and research institutions. DINI will incorporate international developments and ideas, and will co-operate with projects like OpenDOAR, SHERPA (Securing a Hybrid Environment for Research Preservation and Access) and DRIVER (Digital Repository Infrastructure Vision for European Research) (Lossau, 2006). 
Nevertheless, standardisation and interoperability are still badly needed in order to build service layers on top of institutional repositories. The provision of a self-upload tool or self-cataloguing by authors are first steps, access statistics are a key issue for visibility and a step towards alternative impact metrics. DINI's expertise lies in the support and marketing of interoperability technologies for information infrastructures. For this reason, DINI will continue its work on repository certification and will enrich this approach with active partners world-wide in order to launch additional infrastructural activities to promote Open Access. DINI plans to form a core group to develop an Open Access advocacy package, to support the "green way" by integrating more information about German publishers' Open Access policies into SHERPA, to offer advice for authors, librarians, and especially for university management and funding bodies.

On a technical level, DINI plans to enhance interoperability between German Open Access repositories, and to develop an infrastructure to measure impact factors, which takes into account repository content as well as journal content based on internationally agreed standards, technologies and methods.

\section{Notes}

1. www.dini.de

2. www.dini.de/dini/arbeitsgruppe/arbeitsgruppen.php

3. www.dini.de/veranstaltung/workshop/goettingen_2005-05-23/

4. www.dini.de/veranstaltung/workshop/oaimpact/

5. www.longtermpreservation.de

6. www.dini.de/dini/zertifikat/zertifiziert.php (last visited on 14.03.2006)

7. www.persistent-identifier.de

8. http://archives.eprints.org/

9. www.opendoar.org

10. www.sherpa.ac.uk/romeo.php

11. http://w210.ub.uni-tuebingen.de/dbt/uni/licenses.php

12. http://creativecommons.org/

13. www.telesec.de/

14. www.d-trust.net/

15. www.signtrust.de

16. www.kopal.langzeitarchivierung.de/

17. www.bibliothek.uni-regensburg.de/Systematik/systemat.html

18. www.ddb.de/standardisierung/normdateien/swd.htm

19. www.eprints.org

20. www.dspace.org

21. http://elib.uni-stuttgart.de/opus/doku/opus_sw.php

22. www.ddc-deutsch.de/

23. See description at www.editeur.org/

24. www.scirus.com

25. http://base.ub.uni-bielefeld.de/index_english.html 
26. See e.g. www.robotstxt.org/wc/active/all.txt

27. Via Thompson Scientific, Web of Knowledge http://scientific.thomson.com/webofknowledge/aboutwok.html

\section{References}

Erstes Gesetz zur Änderung des Signaturgesetzes (1. SigÄndG) vom 04.01.2005, Bundesgesetzblatt, No. 1 of 10 January 2005.

Ball, P (2006), "Prestige is factored into journal ratings: 'Y-factor' measures quality as well as quantity of citations", Nature, Vol. 439, No. 7078, pp. 770-771.

Bollen, J., Van de Sompel, H. S. JA. and Luce, R. (2005), "Toward alternative metrics of journal impact: a comparison of download and citation data", Information Processing \& Management, Vol. 41, No. 6, pp. 1419-1440.

Brody, T. (2006), Citation Impact of Open Access Articles vs. Articles Available Only Through Subscription ("Toll-Access"), Ongoing study, available at: www.citebase.org/isi_study/ (accessed 27 April 2006).

Consultative Committee for Space Data Systems (CCSDS) (2002), Reference Model for an Open Archival Information System (OAIS), Blue Book, available at: http://public.ccsds.org/publications/archive/650x0b1.pdf (accessed 24 April 2006).

Counting Online Usage of Networked Electronic Resources (COUNTER) (2006), COUNTER Code of Practice for Books and Reference Works Release 1 (March 2006), available at: www.projectcounter.org/code_practice.html (accessed 27 April 2006).

Counting Online Usage of Networked Electronic Resources (COUNTER) (2005), COUNTER code of Practice for Journals and Databases: Release 2 (April 2005), available at: www.projectcounter.org/code_practice.html (accessed 27 April 2006).

Dale, R. L. (2005), "Making certification real: developing methodology for evaluating repository trustworthiness", RLG DigiNews, Vol. 9, No. 5, available at: www.rlg.org/en/page.php?Page_ID=20793\#article2 (accessed 26 April 2006).

Deutsche Initiative für Netzwerkinformation (DINI), Electronic Publishing Working Group (2003), DINI-Certificate for Document and Publication Repositories, available at: www.dini.de/documents/Zertifikat-en.pdf (accessed 27 April 2006).

Deutsche Initiative für Netzwerkinformation (DINI), Electronic Publishing Working Group (2006), DINI-Certificate for Document and Publication Repositories, (in press).

Die Deutsche Bibliothek (2006), LMER: Long-term Preservation Metadata for Electronic Resources, available at: www.ddb.de/standards/pdf/Imer12_e.pdf (accessed 26 April 2006).

Deutsche Initiative für Netzwerkinformation (DINI), Electronic Publishing Working Group (2005), Electronic Publishing in Higher Education: How to design $O A I$ interfaces - Recommendations -, 2nd edition, available at: http://edoc.hu-berlin.de/series/dini-schriften/2005-2-de2/PDF/2-de2.pdf (accessed 27 April 2006). 
Dobratz, S., Neuroth, H., Schoger, A. and Strathmann, S. (2005), "nestor Entwicklungsstand des Kompetenznetzwerks zur Langzeitarchivierung digitaler Ressourcen in Deutschland", ZfBB - Zeitschrift für Bibliothekswesen und Bibliographie, Vol. 52, No. 3-4, pp. 151-162.

Dobratz, S. and Schoger, A. (2005), "Digital repository certification: a report from Germany", RLG DigiNews, Vol. 9, No. 5, available at: www.rlg.org/en/page.php?Page_ID=20793\#article3 (accessed 26 April 2006).

Guédeon, J.-C. (2004), "The "green" and "gold" roads to Open Access: the case for mixing and matching.", Serials Review, Vol. 30, No. 4.

Hardy, R., Oppenheim, C., Brody, T. and Hitchcock, S. (2005), Open Access Citation Information, available at: http://eprints.ecs.soton.ac.uk/11536/ (accessed 27 April 2006).

Harnad, S. (2001), "The self-archiving initiative", Nature, Vol. 410, No. 6832, pp. 1024-1025.

Harnad, S. (2005), "Fast-forward on the green road to Open Access: the case against mixing up green and gold", Ariadne, No. 42, available at: www.ariadne.ac.uk/issue42/harnad/ (accessed 26 April 2006).

Harnad, S., Brody, T., Vallieres, F., Carr, L., Hitchcock, S., Gingras, Y., Stamerjohanns, H. and Hilf, E. (2004), "The access / impact problem and the green and gold roads to Open Access", Serials Review, Vol. 30, No. 4, pp. 310-314.

International Standardisation Organisation (ISO) (2003), Information and documentation - The Dublin Core metadata element set ANSI/NISO Z39.85-20012 or ISO 15836:2003, available at: www.niso.org/international/SC4/n515.pdf (accessed 27 April 2006).

Lagoze, C., Van de Sompel, H. and Nelson, M. W. S. (2002), The Open Archives Protocol for Metadata Harvesting, available at: www.openarchives.org/OAl/openarchivesprotocol.html (accessed 27 April 2006).

Lawrence, S. (2001), "Free online availability substantially increases a paper's impact", Nature, Vol. 411, No. 6837, p. 521.

Lossau, N. (2006), "Digital Repository Infrastructure Vision for European Research", International Workshop on Institutional Repositories and Enhanced and Alternative Metrics of Publication Impact 20-21 February 2006, Berlin, Humboldt University, available at: www.dini.de/veranstaltung/workshop/oaimpact/presentations/lossau.ppt (accessed 27 April 2006).

METS Editorial Board (2005), METS: Metadata Encoding and Transmission Standard: Schema and Documentation, available at: www.loc.gov/standards/mets/ (accessed 27 April 2006).

Mittler, E. and Schulz, M. (2004), "ProPrint world-wide print-on-demand services for study and research", Library Hi Tech, Vol. 22, No. 2, pp. 227-230.

PREMIS Working Group (2005), Data Dictionary for Preservation Metadata, available at: www.oclc.org/research/projects/pmwg/premis-final.pdf (accessed 27 April 2006). 
RLG/NARA Task Force on Digital Repository Certification (2005), Audit Checklist for Certifying Digital Repositories, RLG/NARA Task Force on Digital Repository Certification, Mountain View, CA.

RLG Working Group on Digital Archive Attributes (2002), Trusted Digital Repositories: Attributes and Responsibilities, RLG, OCLC, Mountain View, CA available at: www.rlg.org/legacy/longterm/repositories.pdf (accessed 27 April 2006).

Ross, S. and McHugh, A. (2005), "Audit and certification of digital repositories: creating a mandate for the Digital Curation Centre (DCC)", RLG DigiNews, Vol. $\quad 9, \quad$ No. $5, \quad$ available at: www.rlg.org/en/page.php?Page_ID=20793\#article3 (accessed 26 April 2006).

Schirmbacher, P. (2005), "Die neue Kultur des elektronischen Publizierens," in: Nielsen, E.K., Saur, K.G. and Ceynowa, K. (Eds.), Die innovative Bibliothek, K. G. Saur, Munich, pp. 107-120.

Scholze, F. and Stephan, W. (2002), "Electronic publishing", in: Leonhard, J.-F. (Ed.), Handbücher zur Sprach- und Kommunikationswissenschaft, Vol. 15,3, De Gruyter, Berlin, pp. 2634 - 2648.

Schroeder, K. (2003), "Persistent Identifiers im Kontext von Metadaten", ZfBB Zeitschrift für Bibliothekswesen und Bibliografie, Vol. 50, No. 4, pp. 205209. 